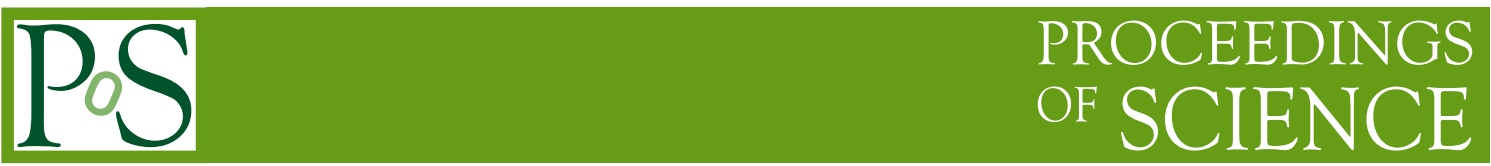

\title{
Physics with low energy antiprotons (antihydrogen)
}

\author{
Germano Bonomi* \\ University of Brescia, via Branze 38, Brescia and INFN Pavia, via Bassi 6, Pavia, Italy \\ E-mail: germano.bonomi@ing.unibs.it
}

\begin{abstract}
The first observations of antihydrogen $(\overline{\mathrm{H}})$ atoms $[1,2]$ in 1995 opened a new way of testing the fundamental symmetries of nature. These first experiments were not suited to precision comparison measurements having produced antihydrogen in small quantities and at high energy. A second generation of experiments was then built with the aim of creating antihydrogen in a controlled process inside an electromagnetic trap. ATHENA and ATRAP indeed succeeded in producing for the first time cold antihydrogen by mixing antiprotons ( $\bar{p}$ 's $)$ and positrons $\left(e^{+}\right.$'s $)$at low temperature (few K) in a nested Penning trap [3, 4]. This result was made possible thanks to the CERN Antiproton Decelerator (AD), the only facility in the world to deliver low energy antiprotons for antihydrogen physics. Since then, upgrades to the experiments were made to keep the antiatoms inside the traps, in order to perform atomic spectroscopy and thus test CPT. With such objective three experiments are at the moment on the floor: ALPHA, ASACUSA and ATRAP. Another experiment, AEgIS, was recently approved and will soon start construction in the AD hall. It aims at directly measuring the gravitational acceleration $g$ on a beam of cold antihydrogen. An overview of past, present and future antihydrogen experimental activities will be given.
\end{abstract}

XLVIII International Winter Meeting on Nuclear Physics, BORMIO2010

January 25-29, 2010

Bormio, Italy

${ }^{*}$ Speaker. 


\section{Introduction}

Antiproton was discovered for the first time in 1955 by E. Segrè and O. Chamberlain; for their discovery they were awarded the Nobel Prize in Physics in 1959. Scientists then tried to study its properties and, many years later, to complement it with an antielectron (positron) to create the simplest atom made with antimatter constituents, the antihydrogen. This article will briefly describe how antimatter is intimately linked to the symmetries of nature and why antihydrogen is the most suitable candidate to test them. After a short experimental history of antihydrogen, the present status and future prospects of CPT and WEP tests will be presented.

\section{Antimatter and the symmetries of nature}

$\Lambda \mathrm{CDM}$, or Lambda-CDM is an abbreviation for Lambda-Cold Dark Matter. It is frequently referred to as the standard model of big bang cosmology. It is the simplest model that is in general agreement with observed phenomena, such as the existence and structure of the cosmic microwave background, the large scale structure of galaxy clusters and the distribution of light elements and the accelerating expansion of the Universe observed in the light from distant galaxies and supernovae. This standard model works with two unknown entities that constitute about $95 \%$ of the Universe composition: the dark matter (25\%) and the dark energy (70\%). In other words, we know almost everything about the history of the Universe, but we ignore $95 \%$ of its nature.

A. Benoit-Lévy and G. Chardin are proposing an alternative and unconventional model, the so-called "Dirac-Milne" Universe. It portrays a matter-antimatter symmetric cosmology [5, 6], in which the antimatter originated in the big bang would still be around. This simple Universe seems to satisfy: (1) the cosmological tests for the age of the Universe, (2) the big bang nucleosynthesis, (3) the Type Ia Supernovae data and (4) the "acoustic peak" of the Cosmological Microwave Background. Surely it represents an intriguing point of view, specially because it requires a gravitational repulsion between matter and antimatter. Independently of the reliability of such model undoubtedly antimatter is intimately connected with the history (and composition) of the Universe: antimatter is thus an ideal "test-bed" for studying the (a)symmetries of nature.

A fundamental one is summarized in the so-called CPT theorem according to which the laws of nature are symmetric under the inversions of charge (C), parity (P) and time (T). Since CPT transform an elementary particle into its antiparticle, this predestines antimatter for tests of such invariance. Various experiments have been performed comparing different properties, such as the magnetic moment, the charge to mass ratio and the mass, of a particle and of its antiparticle. The best limits are summarized in fig. 1. In particular from the comparison of the gyromagnetic moment of $e^{-}$and $e^{+}$a limit of $10^{-12}$ could be deduced in terms of relative precision, while the $K^{o}-\bar{K}^{o}$ mass difference sets a limit of $10^{-18}$. However, according to some theoretician both measurements are model dependent. Indeed in specific models CPT may be broken without affecting the gyromagnetic moment [7, 8] while the limit extracted from the $K^{o}-\bar{K}^{o}$ mass difference [9] assumes the validity of the standard model which does not contain itself a mechanism to violate CPT. On the other hand, differently from a single antiparticle, antihydrogen is a complete system perfectly suitable for a direct test of CPT. Using the same experimental methods of hydrogen spectroscopy high precision comparison of the two system may be achieved. Indeed antihydrogen is a mostly 


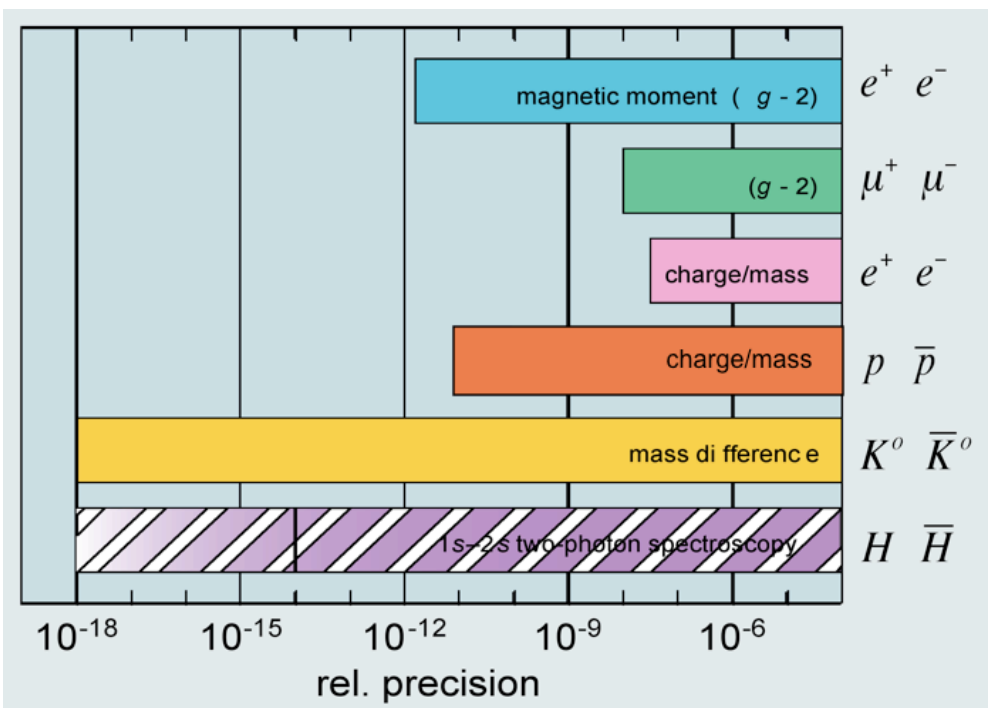

Figure 1: Relative precision of various CPT tests performed comparing different properties of a particle and its antiparticle counterpart.

electromagnetic system (weak interaction, or parity violating, effects are small and the same for hydrogen and antihydrogen) making the comparison matter-antimatter less model dependent.

Another important symmetry of nature is enclosed in the Weak Equivalence Principle (WEP), expressed in Einstein general relativity theory, that states that the gravitational acceleration of a falling object is independent of its composition. Indeed composite objects of ordinary matter have shown equal gravitational acceleration to one part in $10^{12}$. According to this principle it should also be the same for matter and antimatter. Even if arguments against "antigravity" have been given by numerous authors [10] and the majority of the scientific community gives poor credit to a different scenario, no experimental test of the WEP has been performed with particles and antiparticles. The hypothesis of a repulsion between matter and antimatter, as previously reported, would also imply a strong revision to our cosmological knowledge. Some experiments have been proposed to measure the falling acceleration of charged antiparticles, but due to the difficulties in shielding electromagnetic effects, much higher than the gravitational ones, no results have been achieved. On the other hand antihydrogen is a neutral system free from problems associated with electromagnetic interactions and consequently is perfect for a direct test of the gravitational interaction of antimatter with the Earth field.

Summarizing, antihydrogen is the ideal candidate to study the symmetries of nature, better than antiprotons or positrons alone. Are we able to produce it and to handle it? It took few decades from the discovery of the antiprotons, but finally at the end of the last millenium the first antiatoms were created.

\section{The history of antihydrogen and status of CPT tests}

To produce antihydrogen from its constituents, antiprotons and positrons are needed. If it is relatively easy to obtain positrons from $\left(\beta^{+}\right)$radioactive decays, much more difficult is the creation of 


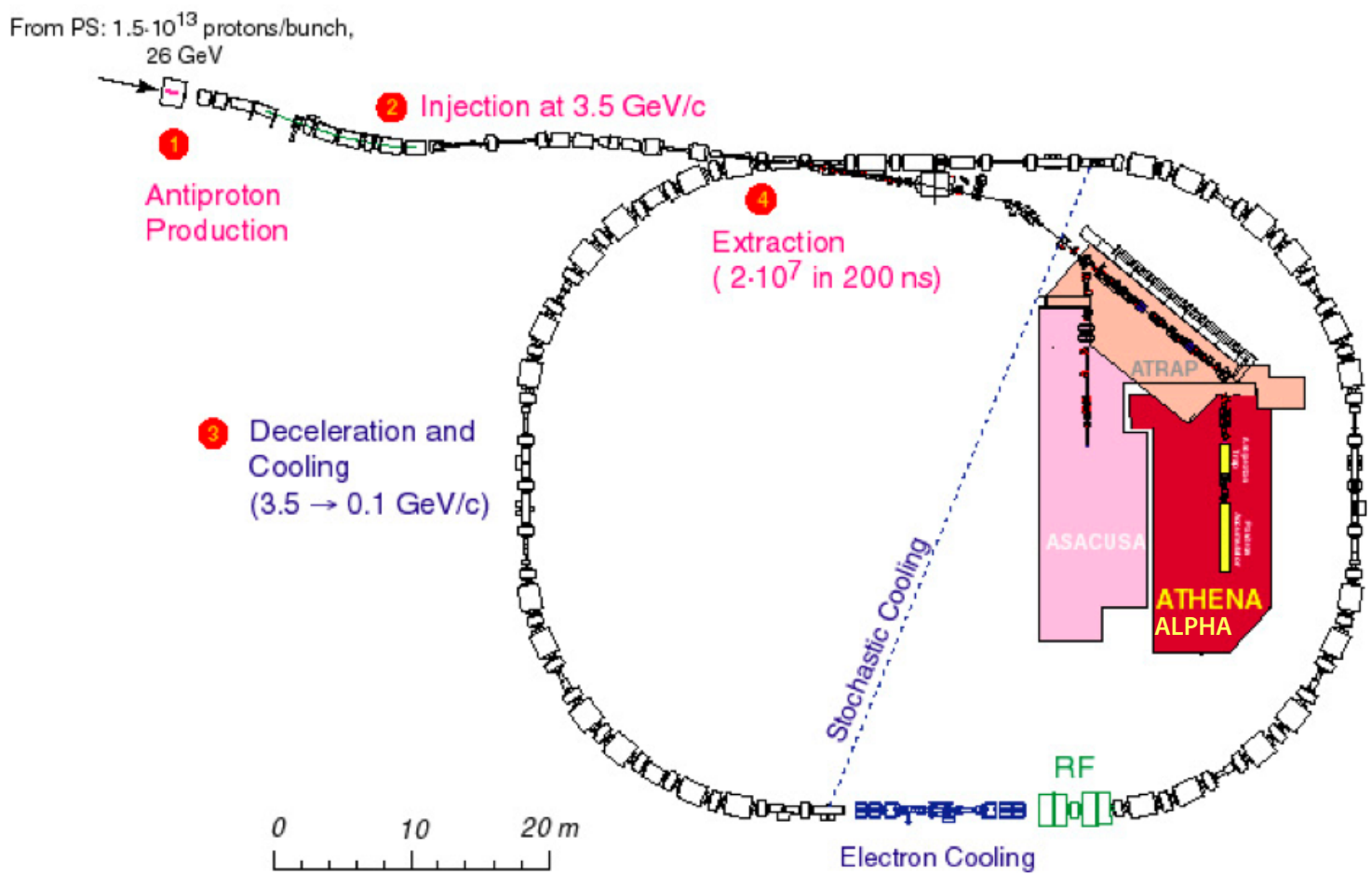

Figure 2: Schematic overview of the AD (Antiproton Decelerator) hall at CERN.

a usable sample of antiprotons. Indeed the history of antihydrogen started with the construction of LEAR machine at CERN. In the 90', the Low Energy Antiprotons Ring could deliver "low energy" $(1.2 \mathrm{GeV} / \mathrm{c}$ of momentum) antiprotons to various experiments. One of them, PS210, exploited the interaction between the antiprotons and jets of Xenon atoms. With a relative probability of $10^{19}$ a particular reaction could give birth to an antihydrogen. The experiment measured 9 events, [1], the first antihydrogen atoms ever produced. In the following years a similar result was obtained at Fermilab with a final sample of about 100 events [2]. This way of producing antihydrogen was clearly low efficient $\left(1 \overline{\mathrm{H}}\right.$ per $\left.10^{19} \bar{p}\right)$ and, since the antiprotons were let to fly through a Xenon jet, the antihydrogen were too fast to make them usable for any further study.

When LEAR ended its operation, the "low energy" antiproton physics community pushed for a new machine able to deliver even lower energy $\bar{p}$ 's. From the ashes of LEAR, at CERN, the $\mathrm{AD}$ machine (Antiproton Decelerator) rised (see fig. 2). The $26 \mathrm{GeV}$ proton beam (about $1.510^{13}$ protons/bunch) from the PS impacts a production target from which the antiprotons are extracted and injected at $3.5 \mathrm{GeV} / \mathrm{c}$ inside the $\mathrm{AD}$ decelerating ring. In this way around $2 \times 10^{7}$ antiprotons every $\sim 100 \mathrm{~s}$, with a momentum of $100 \mathrm{MeV} / \mathrm{c}(5.3 \mathrm{MeV}$ in kinetic energy), were at hand for antimatter physics. Thanks to the availability of such a $\bar{p}$ beam, a $2^{\text {nd }}$ generation of antihydrogen experiments was planned and built: ATHENA and ATRAP. The specific goal was the creation of antihydrogen in electro-magnetic traps for future spectroscopy studies. In the AD hall, in the same period (around the year 2000), another experiment ASACUSA was also starting operation for the study of the properties of the antiprotonic-helium. Being different on the experimental apparatus 


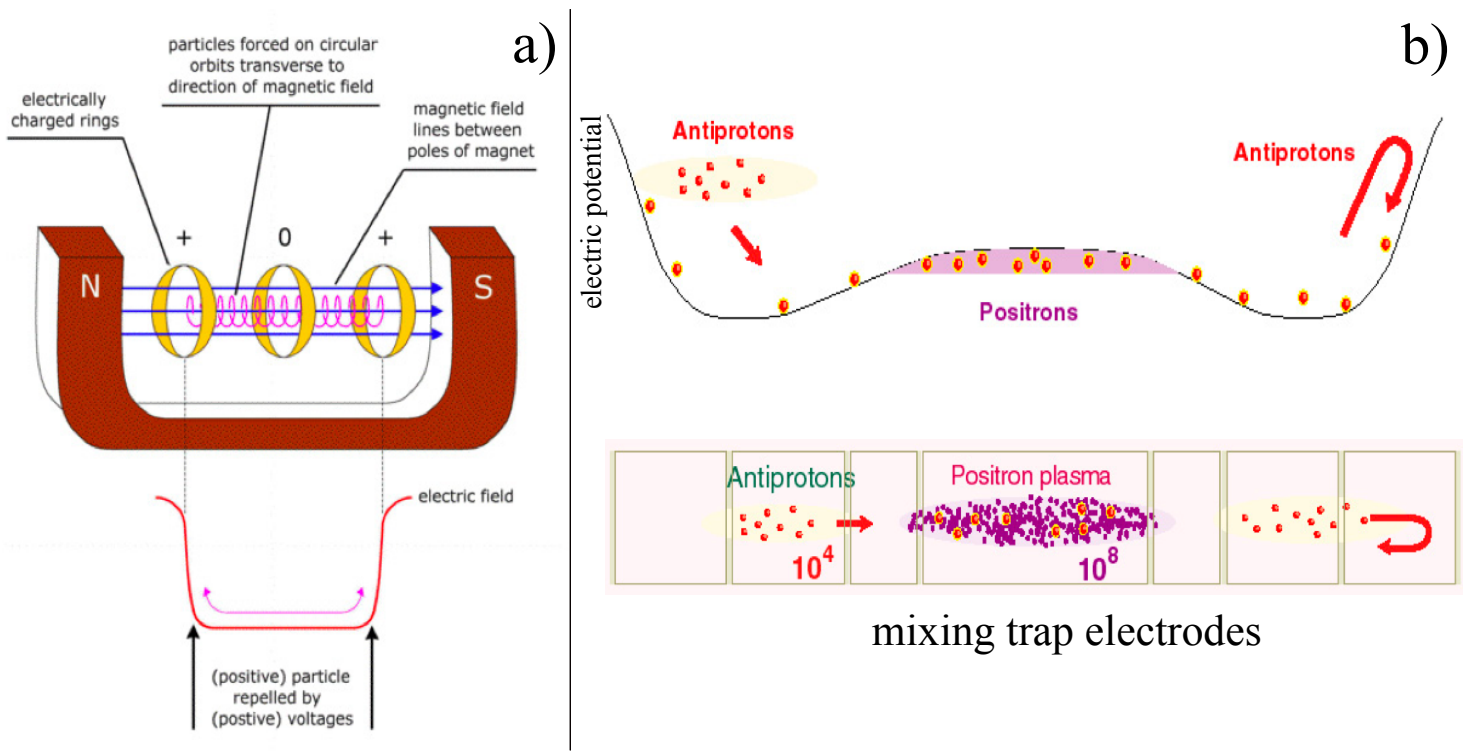

Figure 3: a) Schematic view of an electromagnetic trap. b) Schematic view of the so called "nested Penning trap" [11].

the basic concept of both ATHENA and ATRAP was the following: (1) collect antiprotons from the $\mathrm{AD}$ and positrons from a ${ }^{22} \mathrm{Na}$ radioactive source, (2) store them separately using trap electrodes voltages (fig. 3a) to confine the charged particles axially and a magnetic field to confine them radially, (3) mix them inside a so called "nested Penning trap" (fig. 3b), and (4) monitor the blending to discover evidence of antihydrogen atoms. In particular when antihydrogen is formed, being neutral, it escapes the mixing region and it annihilates on the trap walls. A space-time coincidence of antiproton and positron annihilations is thus a clear sign of production.

In 2002 ATHENA first, and ATRAP few months later, indeed reported the first creation of "cold" antihydrogen [3,4]. Antiatoms were finally avaibale in traps. In a sense it was like having a micro antiworld in a small cage. Unfortunately such antiatoms were escaping the trap as soon as they were produced, so they were unusable for any further study. They needed to be confined before trying to investigate them with lasers for spectroscopy for a CPT test. In 2005 and 2006 $\mathrm{AD}$ was shut down in a saving plan for the LHC. It was the right time for an upgrade. The place of ATHENA was taken by a new experiment, ALPHA, while the ASACUSA collaboration also added to its goals antihydrogen physics. The "next step" for these experiments was to try confine both charged particles and neutral atoms in the same region. For plasma confinement the cylindrical symmetry has to be preserved while for atom confinement the magnetic field must be minimal in the center. The solution proposed by ALPHA and ATRAP was to superpose a radial magnetic multiple trap upon a solenoidal field. ASACUSA on the other hand decided to avoid difficult trapping and intend to make an antihydrogen beam, since it can count on a Radio Frequency Quadruple Decelerator (RFQD) able to further decelerate the AD antiproton beam down to $50 \mathrm{keV}$. To date, no trapping or beam production, has been reported. A possible problem for confinement is that the antihydrogen seems too "hot" (tens or hundreds of K) to be trapped. For example the ALPHA 

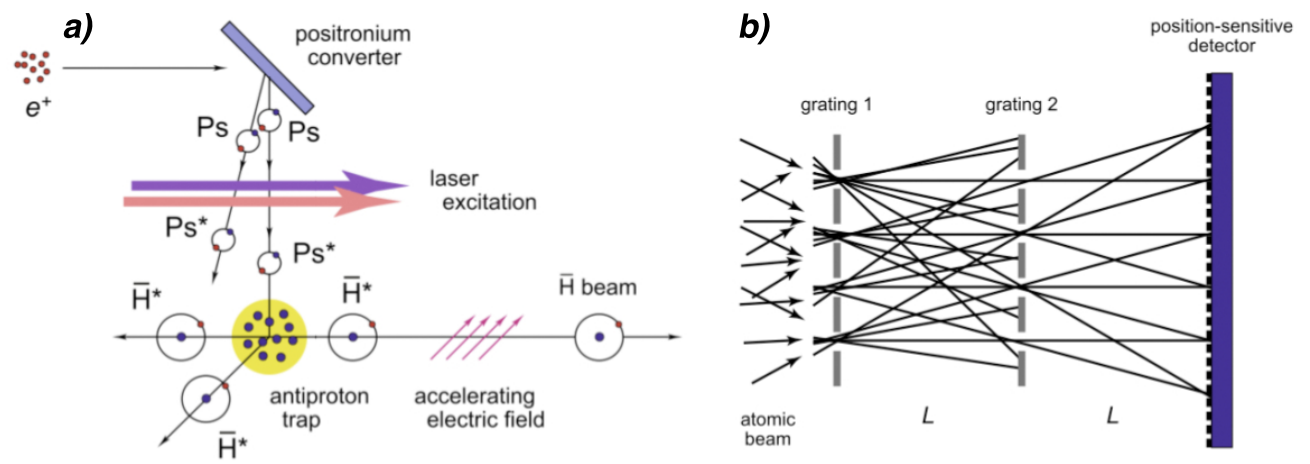

Figure 4: (a) AE $\bar{g} I S$ scheme for $\overline{\mathrm{H}}$ beam formation; (b) configuration of the Moiré deflectometer.

magnetic trap for neutrals is $0.5 \mathrm{~K}$ deep ${ }^{1}$. As far as experiments stand, the CPT test (spectroscopy) with antihydrogen seems not yet feasible, at least in the very near future. In the next years (the AD machine operation has been approved and financed up to 2016) the three experiments on the floor, will try hard to achieve trapping, or beam production, to perform the first spectroscopic measurements. In particular ATRAP and ALPHA aims at measuring the 1S-2S transition (see [12] for a possible scheme) while ASACUSA intend to carry out microwave spectroscopy of the ground-state hyperfine structure [13].

\section{On the way to test WEP - The AEgIS experiment}

Gravitational interaction between matter and antimatter has never been tested experimentally. As previously reported although most of the theoretical predictions suggest that antimatter should behave like matter in a gravitational field such as the Earth's, other possibilities are still open (see for example the captivating model by Chardin and collaborators [5]). In 2007 a new collaboration submitted at the CERN SPSC (SPS experiments Committee) a proposal [14] for a new experiment, called AEgIS (Antimatter Experiment: Gravity, Interferometry, Spectroscopy). The primary scientific goal is to perform, for the first time, a direct measurement of the gravitational acceleration $g$ on a beam of antihydrogen with a $1 \%$ relative precision. The measurement will be carried out by observing the vertical displacement of the shadow image produced by an $\overline{\mathrm{H}}$ beam as it traverses a Moiré deflectometer (see below). The overall scheme of $\overline{\mathrm{H}}$ production and beam formation is summarized in Fig. 4a, while the details of the whole process are described thoroughly in the experiment proposal [14]. For what concerns the production mechanism the experiment will rely on the so called resonant charge exchange reaction $\mathrm{Ps}^{*}+\bar{p} \rightarrow \overline{\mathrm{H}}^{*}+e^{-}[15,16]$, where Ps stands for Positronium and the star denotes a highly excited Rydberg state. The cross-section scales approximately with the fourth power of the principal quantum number of the Positronium $\left(n_{\mathrm{P}_{\mathrm{s}}}\right)$, therefore, tuning $n_{\mathrm{P}_{\mathrm{s}}}$, high rates can be achieved. Antihydrogen is also created in a narrow and well-defined band of final states ( $n_{\overline{\mathrm{H}}} \simeq \sqrt{2} n_{\mathrm{P}_{\mathrm{s}}}$, with a rms of few units). When formed with antiprotons at rest, the antihydrogen is generated with a velocity distribution dominated by the antiproton temperature,

\footnotetext{
${ }^{1}$ The energy of antihydrogen is often reported in terms of Kelvin instead of eV. The relation between them is expressed by the formula $\mathrm{E}=\mathrm{kT}$, where $\mathrm{k}$ is the Boltzmann constant. For example $1 \mathrm{meV}$ correspont to $11.6 \mathrm{~K}$.
} 
thus, in principle, very cold antihydrogen can be formed. Once the production has taken place, the resulting antiatoms will be then accelerated by means of inhomogeneous electric fields, with a method called "Stark acceleration" [17, 18]. A more comprehensive description of the antihydrogen beam formation can be found in [19]. This pulsed production technique provides the starting time for the gravity measurements and offers the possibility to easily measure, by time of flight, the resulting antihydrogen temperature. When created the beam freely falls in the Earth's gravitational field. Given AEḡIS realistic numbers, flight path length of $1 \mathrm{~m}$, horizontal velocity of about 500 $\mathrm{m} / \mathrm{s}$, the vertical displacement of an antihydrogen atom due to gravity, assuming $g \sim 10 \mathrm{~m} / \mathrm{s}$, would be about $20 \mu \mathrm{m}$. However, when created the antihydrogen has a thermal velocity in all directions of a few tens of $\mathrm{m} / \mathrm{s}$. During the flight path an antihydrogen atom can thus displace vertically up to $10 \mathrm{~cm}$, and it is not possible, for each antiatom, to know its radial velocity. Clearly it is impossible to measure the gravitational acceleration $g$, simply measuring the vertical displacement. That's the reason why the collaboration proposed to use a Moiré deflectometer (fig. 4b), already applied to measure the gravitational acceleration of a beam of (ordinary matter) Argon atoms [20]. The system is based on geometric propagation of an (anti)atomic beam through a set of identical gratings. The first two gratings select propagation directions of an originally diverging atomic beam. Beyond such gratings, at a distance equal to the distance between the first two, the atoms are distributed in a shadow image forming sets of fringes. The method also works with non-collimated beams characterized by a broad energy distribution, such as the AEḡIS one. In its original form it consisted of three material gratings, equally spaced and aligned parallel to each other, while in the AE $\bar{g} I S$ configuration (see fig. $4 \mathrm{~b}$ ) the third grating is replaced by a position-sensitive annihilation detector to measure the $\overline{\mathrm{H}}$ annihilation point. In this way the set of fringes will be measured as annihilation density variations. Due to the gravitational fall, the maximum of the fringe would not be aligned with the grating openings, but would suffer a shift equal to $\delta=-\mathrm{g} T^{2}$, where $T$ is the time of flight between two adjacent gratings. This time can be calculated since both the starting time (Stark acceleration) and the stopping time (annihilation) are known with a precision much smaller than the typical time of flight of few ms. From the measurement of the fringe shift $\delta$, the acceleration constant $g$ can thus be obtained. The AEgIS experiment is now finalizing the overall design and starting the construction. Its physics program will span up to 2016 and it will accompany the ALPHA, ASACUSA and ATRAP ones. It is important to remind here that the AD antiproton beam has been also used to test the clinical potentials of antiprotons for the cure of tumors [21].

\section{Future prospects and conclusions}

Antihydrogen is surely essential to study fundamental properties of nature and great technological steps have been made since the discovery of the first antiatoms. In a sense the way is marked but other steps have still to be made, and every one is extremely challenging. The next years will be important to finally compare the properties of atoms and antiatoms.

As reported previously the AD machine has been approved until 2016. Possibly it will be improved by the construction of the so-called ELENA (Extra Low ENergy Antiproton ring) ring, that will bring the energy of the antiprotons further down to $0.1 \mathrm{MeV}$ [22] rendering the creation of antihydrogen much more efficient. After 2016 the future of the antiproton/antihydrogen physics 
community may move to GSI, where a specific low-energy $(0.3 \mathrm{MeV})$ antiproton beam is foreseen inside the FAIR facility (Facility for Antiproton and Ion Research).

\section{References}

[1] Baur, G. et al., Production of antihydrogen. Phys. Lett. B 368, $251 Đ 258$ (1996).

[2] Blanford, G. et al., Observation of atomic antihydrogen. Phys. Rev. Lett. 80, 3037Đ3040 (1998).

[3] M. Amoretti, et al., Nature 419 (2002) 456.

[4] G. Gabrielse, et al., Phys. Rev. Lett. 89 (2002) 213401.

[5] Aurélien Benoit-Lévy and Gabriel Chardin, arXiv: 0903.2446v1 [astro.ph-CO] 13 Mar 2009.

[6] Mathieu Grousson, Science et Vie, Antimatière. Est-elle la clé de l'Univers?, N. 1105, Octobre 2009.

[7] R. Bluhm, V. A. Kostelecky, N. Russell, Phys. Rev. Lett. 79 (1997) 1432.

[8] D. Colladay and V.A. Kostelecky Phys. Rev. D 55 (1997) 6760.

[9] E. Shabalin, Phys. At. Nuclei 57 (1994) 1862.

[10] M. M. Nieto and T. Goldman, Phys. Rep. 205 (1991) 221.

[11] G. Gabrielse et al. Phys.Lett. A129, 38 (1988).

[12] J. Walz et al., Hyp. Int. 127 (2000) 167.

[13] ASACUSA collaboration, Addendum to the proposal CERN/SPSC 97-19 and CERN/SPSC 2000-04, CERN-SPSC 2005-002/SPSC P-307 Add.1.

[14] G. Drobychev et al. CERN-SPSC-2007-017, http://cdsweb.cern.ch/record/1037532 (2007).

[15] M. Charlton, Phys. Lett. A 143 (1990) 143.

[16] C. Storry et al., Phys. Rev. Lett. 93 (2004) 263401.

[17] E. Vliegen, F. Merkt, J. Phys. B 39 (2006) L241.

[18] E. Vliegen et al., Phys. Rev. A 76 (2007) 023405.

[19] G. Testera et al., Proceeding of Cold Antimatter Plasmas and Application to Fundamental Physics Conference, AIP Conference Proceedings, 1037 (2008) 5.

[20] M. K. Oberthaler et al., Phys Rev A 54 (1996) 3165.

[21] N. Bassler and M. Holzscheiter, Acta Oncologica 48 (2009) 223 and references therein.

[22] T. Eriksson, Hyperfine Interact 194 (2009) 123. 